\title{
Gravidez Ectópica após Uso de Contracepcão de Emergência: Relato de Caso
}

\author{
Ectopic Pregnancy after Use of Hormonal Emergency Contraception: a Case Report
}

Renato Monteiro Zucchi, Julio Elito Jr, Flavio Zucchi, Luiz Camano

\section{RESUMO}

\begin{abstract}
Gravidez ectópica é a implantação e o desenvolvimento do ovo fora da cavidade uterina; impõe diagnóstico precoce e assistência de urgência. Gravidez ectópica com corpo lúteo contralateral presume ocorrência de transmigração do zigoto para a tuba uterina do lado oposto, o que pode ser responsável pela sua ocorrência. Em 1994, o levonorgestrel teve sua eficácia comprovada como superior à dos outros métodos para contracepção de emergência. É relatado caso de paciente de 27 anos que apresentou gravidez ectópica, de localização tubária, com corpo lúteo contralateral, após uso de contracepção de emergência por falha do método anticoncepcional de escolha (preservativo masculino). Foi realizado tratamento cirúrgico conservador por videolaparoscopia, com boa evolução após a cirurgia.
\end{abstract}

PALAVRAS-CHAVE: Gravidez ectópica. Anticoncepção. Videolaparoscopia.

\section{Introdução}

Gravidez ectópica (GE) é a implantação e o desenvolvimento do ovo fora da grande cavidade uterina. É uma hemorragia do primeiro trimestre da gestação, podendo se manifestar com quadro abdominal agudo, que impõe diagnóstico precoce e assistência de urgência. A localização tubária é a mais freqüente, ocorrendo em torno de $95 \%$ dos casos, e sua incidência vem crescendo, chegando a 1 para cada 80 a 100 gestações $^{1-3}$.

As principais causas de GE são: salpingites, cirurgias tubárias prévias, alterações anatômicas, endometriose, tumorações justatubárias, alterações da motilidade tubária (em virtude de práticas contraceptivas) e uso de DIU, entre outras ${ }^{1}$.

Gravidez ectópica com presença de corpo lúteo contralateral presume ocorrência de transmigração do zigoto para a tuba uterina do

Departamento de Obstetrícia da Universidade Federal de São Paulo - Escola Paulista de Medicina

Correspondência:

Renato Monteiro Zucchi

Rua Morgado de Mateus, 314 - ap. 182

04015-050 - São Paulo - SP

Tel: (11) 5579-9350 / 9402-1027

Fax: (11) 5539-3176

e-mail: renatozucchi@hotmail.com lado oposto. Como não se sabe ao certo a incidência de tal transmigração em gestações tópicas, presume-se que a sua ocorrência esteja diretamente ligada à GE, devido ao atraso na chegada do ovo ao útero, por percorrer caminho mais longo do que o normal ${ }^{4}$.

Os métodos de contracepção de emergência foram desenvolvidos e são eficazes no caso de relações sexuais desprotegidas no meio do ciclo menstrual $^{5}$. O levonorgestrel 0,75 mg foi lançado em 1979, inicialmente em alguns países. Ressalta-se, contudo, que somente em 1994, após estudo clínico realizado pela OMS, sua eficácia foi comprovada como superior à dos outros métodos de contracepção de emergência existentes até então $0^{6,7}$.

O levonorgestrel é capaz de realizar a contracepção de emergência por meio de diversos mecanismos de ação. Dentre eles podem ser citados as alterações: na capacidade de fecundação dos espermatozóides, na ovulação (caso a droga tenha sido tomada antes da sua ocorrência), na formação do corpo lúteo, no endométrio, no muco cervical e na motilidade tubária por inversão do peristaltismo e do batimento das fímbrias ${ }^{8-15}$.

O objetivo deste trabalho é fazer o relato da ocorrência de gravidez ectópica com corpo lúteo contralateral após uso de contracepção de emergência. Alguns aspectos chamam atenção neste 
caso: primeiro, a gravidez após o uso de tal método é pouco freqüente; segundo, a ocorrência de gravidez ectópica, a qual já tem incidência baixa, ao redor de 1\%; e terceiro, a presença de corpo lúteo contralateral, que ocorre em apenas $25 \%$ dos casos de gravidez ectópica.

\section{Relato do Caso}

Paciente KES, 27 anos, procurou atendimento médico, no dia 14 de outubro de 2003 , referindo falha do método contraceptivo de escolha (preservativo masculino), em relação sexual no dia 12 de outubro. Informou ainda ter tido o último episódio menstrual em 29 de setembro de 2003, o que a levou a fazer uso de levonorgestrel 0,75 mg (em duas tomadas com intervalo de 12 horas), pois estava no $14^{\circ}$ dia do ciclo menstrual. Nessa ocasião foi orientada a aguardar o fluxo menstrual. Em 26 de outubro iniciou sangramento genital que persistiu, com aumento progressivo de sua quantidade, até 5 de novembro, quando procurou novamente serviço médico em razão de quadro de dor na fossa ilíaca direita. Ao exame físico apresentava-se em bom estado geral, corada, hidratada e normotensa. O abdômen era plano, flácido, doloroso à palpação profunda na fossa ilíaca direita, a descompressão brusca não era dolorosa e os ruídos hidroaéreos estavam presentes. Ao toque vaginal observou-se dor no fórnice vaginal direito e ao exame combinado, aumento do tamanho do anexo direito com dor. Diante deste quadro clínico foram solicitados exames laboratoriais de $\beta$-hCG sérico quantitativo e ultra-sonografia transvaginal.

O valor do $\beta$-hCG foi de $778,70 \mathrm{mUI} / \mathrm{mL}$ e a ultra-sonografia transvaginal revelou cavidade uterina vazia, ovários direito e esquerdo normais e presença de massa na região anexial à direita (hematossalpinge), medindo $5,5 \mathrm{~cm}$ (longitudinal) x 3,2 cm (transversal) x 2,6 cm (ântero-posterior). Por esses resultados, em associação com a história clínica e o exame físico, foi indicada a videolaparoscopia de urgência.

Após incisão infra-umbilical a céu aberto, introdução da óptica e insuflação da cavidade peritoneal, foi visualizada massa anexial na região ampolar da tuba uterina direita e presença de corpo lúteo no ovário esquerdo. Foi realizada salpingotomia linear no ponto de maior abaulamento da tuba com pinça monopolar, e hidrodissecção seguida da retirada do tecido trofoblástico; a incisão não foi suturada, sendo fechada por segunda intenção. Posterior estudo anatomopatológico do material retirado demonstrou presença de restos ovulares e de decídua parcialmente necróticos. A paciente evo- luiu sem intercorrências no pós-operatório, com melhora da sintomatologia e redução dos níveis séricos de $\beta$-hCG.

\section{Discussão}

O uso de contraceptivos orais de emergência pode ser considerado método seguro para evitar gravidez indesejada após relações sexuais desprotegidas no meio do ciclo menstrual ${ }^{5,16,17}$. Trussell et al. ${ }^{16}$ encontraram 5,2\% de gravidez em estudo clínico realizado com pacientes que fizeram o uso correto de anticoncepção de emergência (levonorgestrel 0,75 mg em duas tomadas com intervalo de 12 horas em no máximo 72 horas após o coito) após relação sexual no período provável da ovulação. Wellbery ${ }^{18}$ relatou redução de $75 \%$ no número de gestações indesejadas com o uso deste método.

Contudo, Farkas ${ }^{7}$ referiu não ser este método seguro, pois observou $6,4 \%$ de gravidez extrauterina, além da alta freqüência de sangramento irregular. Sheffer-Mimouni et al. ${ }^{19}$ relataram três casos de gravidez ectópica com o uso de levonorgestrel próximo ao período ovulatório, e alertaram que profissionais de saúde devem estar atentos para a possibilidade de gravidez ectópica na presença de queixa de dor abdominal após o uso de levonorgestrel.

É provável que a ocorrência de gravidez ectópica esteja diretamente ligada aos mecanismos de ação do levonorgestrel, que ainda não foram totalmente elucidados. Dependendo da proximidade de seu uso com o pico de LH, levonorgestrel pode adiar ou até mesmo inibir a rotura folicular, ou ainda interferir com a formação e com a função do corpo lúteo ${ }^{9,20,21}$. Além disso, esta droga é capaz de alterar a motilidade dos espermatozóides, por aumentar o $\mathrm{pH}$ do fluido uterino e a viscosidade do muco cervical ${ }^{8}$. Alguns autores defendem que o levonorgestrel também possui efeito sobre os eventos que ocorrem após a fecundação, por interferir no preparo do endométrio de algumas moléculas dependentes da progesterona ${ }^{8}$, resultando em meio desfavorável para a implantação do ovo ${ }^{10}$. Outro mecanismo de ação atribuído ao levonorgestrel é a alteração da motilidade tubária, o que impediria a captação e o transporte do óvulo pela tuba em direção ao útero ${ }^{9}$. Sugerimos que este último mecanismo de ação seja responsável pela ocorrência de transmigração do zigoto para a tuba contralateral, com conseqüente gestação ectópica por atraso de sua chegada à cavidade uterina.

No caso em questão optou-se por realizar o 
tratamento cirúrgico conservador, pelo fato de a paciente desejar preservar a fertilidade e pela gravidez ectópica estar íntegra. A videolaparoscopia foi escolhida por representar menor agressão cirúrgica, menor risco de formar aderências e pela melhor recuperação da paciente no pós-operatório ${ }^{2,3}$.

Conclui-se que, apesar de os métodos contraceptivos de emergência terem boa eficácia no caso de relações sexuais desprotegidas no meio do ciclo menstrual, após o seu uso o médico deve sempre estar atento para identificar precocemente os sinais de gravidez ectópica, antecipando o seu diagnóstico e melhorando, assim, a sua condução terapêutica.

\section{ABSTRACT}

Ectopic pregnancy is the implantation and development of the ovum outside the uterine cavity; it needs a quick diagnosis and an urgent treatment. The presence of the corpus luteum in the ovary that is contralateral to the ectopic pregnancy is presumptive evidence for ovum transmigration, which may be the cause of ectopic pregnancy. In 1994, a multinational clinical trial proved the superiority of levonorgestrel over the existing emergency contraceptive products. In the present study, we describe the case of a 27year-old woman with ectopic pregnancy and a contralateral corpus luteum after use of hormonal emergency contraception (levonorgestrel), because of failure of the used contraception method (condom). The patient was treated with laparoscopic surgery that was successful.

KEYWORDS: Ectopic pregnancy. Contraception. Laparoscopy.

\section{Referências}

1. Neme B. Prenhez ectópica. In: Neme B, editor. Obstetrícia Básica. 1a ed. São Paulo: Sarvier; 1994. p. 413-5.

2. Camano L, Elito Junior J, Han KK. Gravidez ectópica. In: Prado FC, Ramos J, Valle JR, editores. Atualização Terapêutica. $21^{\mathrm{a}}$ ed. São Paulo: Artes Médicas; 2003. p. 1026-30.

3. Elito J Jr, Reichmann A, Uchiyama M, Camano L. Predictive score for the systemic treatment of unruptured ectopic pregnancy with a single dose of methotrexate. Int J Gynaecol Obstet 1999; 67:75-9.

4. Ziel HK, Paulson RJ. Contralateral corpus luteum in ectopic pregnancy: what does it tell us about ovum pickup? Fertil Steril 2002; 77:850-1.

5. Sudik R. Extrauterine pregnancy after hormonal postcoital contraception. Zentralbl Gynakol 1995; 117:320-2.
6. Thaler G. Collaboration with academia in the development of post ovulatory methods. Int J Gynaecol Obstet 1999; 67(Suppl 2): S77-83.

7. Farkas M. The effect of oral ovulation inhibiting (mono-, bi-, and triphase) contraceptives and of Continuin and Postinor on proteo- and steroid hormones. Acta Pharm Hung 1992; 62:17-30.

8. Croxatto HB, Ortiz ME, Müller AL. Mechanisms of action of emergency contraception. Steroids 2003; 68:1095-8.

9. Durand M, del Carmen Cravioto M, Raymond EG, et al. On the mechanisms of action of short-term levonorgestrel administration in emergency contraception. Contraception 2001; 64:227-34.

10.Brache V, Faundes A, Johansson E, Alvarez F. Anovulation, inadequate luteal phase and poor sperm penetration in cervical mucus during prolonged use of Norplant implants. Contraception 1985; 31:261-73.

11.Faundes A, Alvarez-Sanchez F, Brache V, Jiménez E, Tejada AS. Hormonal changes associated with bleeding during low dose progestogen contraception delivered by Norplant subdermal implants. Adv Contracept 1991; 7:85-94.

12.Schwarz UI, Buschel B, Kirch W. Unwanted pregnancy on self-medication with St. John's wort despite hormonal contraception. Br J Clin Pharmacol 2003; 55:112-3.

13.Darney PD, Monroe SE, Klaisle CM, Alvarado A. Clinical evaluation of the Capronor contraceptive implant: preliminary report. Am J Obstet Gynecol 1989; 160:1292-5.

14. Croxatto HB, Diaz S, Salvatierra AM, Morales P, Ebensperger C, Brandeis A. Treatment with Norplant subdermal implants inhibits sperm penetration through cervical mucus in vitro. Contraception 1987; 36: 193-201.

15. Facts about implantable contraceptive: memorandum from a WHO meeting. Bull World Health Organ 1985; 63:485-94.

16.Trussell J, Ellertson C, von Hertzen H, et al. Estimating the effectiveness of emergency contraceptive pills. Contraception 2003; 67:259-65.

17.Camp S. Postinor - the unique method of emergency contraception developed in Hungary. Plan Parent Eur 1995; 24:23-4.

18.Wellbery C. Emergency contraception. Arch Fam Med 2000; 9:642-6.

19.Sheffer-Mimouni G, Pauzner D, Maslovitch S, Lessing JB, Gamzu R. Ectopic pregnancies following emergency levonorgestrel contraception. Contraception 2003; 67:267-9.

20.Hapangama D, Glasier AF, Baird DT. The effects of peri-ovulatory administration of levonorgestrel on the menstrual cycles. Contraception 2001; 63:123-9.

21.Marions L, Hultenby K, Lindell I, Sun X, Stabi B, Gemzell Danielsson K. Emergency contraception with mifepristone and levonorgestrel: mechanism of action. Obstet Gynecol 2002; 100:65-71.

Recebido em: 21/5/2004

Aceito com modificações em: 23/8/2004 